\title{
HEARING TESTS IN A RESIDENTIAL UNIT FOR LEARNING-DISABLED PEOPLE
}

\author{
Claire Lewis, Michael Brown, Craig Jackson, Janet Theobold \\ Audiology Department, Queen Victoria Hospital, Morecambe
}

\section{ABSTRACT}

We report on the findings of a survey performed on the hearing and aural health of the residents of a unit for those with learning disabilities.

The results indicate a number of significant findings, the most important of which is the very high prevalence of impacted wax (78\%). In addition, $9 \%$ of the subjects were found to need referral to an ENT surgeon due to lesions within the ear.

During our investigations we were also able to uncover 24 (34\%) previously unreported hearing losses, from mild to profound. The implications of these findings and others are discussed and recommendations made for those thinking of offering a similar service.

\section{INTRODUCTION}

Since the introduction of 'Care in the Community'(1), much more attention has been paid to those who are living in longstay hospital accommodation, and to the development of care programmes to enable them to live within the community, sometimes in a sheltered environment, or with support. Such a programme was initiated at the Royal Albert Hospital (RAH) in Lancaster, which was a residential hospital for learning-disabled people of many ages, backgrounds and capabilities. In 1992, following consultations, a decision was made to test the hearing of all those who were to be discharged into the community so that any with ear pathology or hearing deficit could be identified and appropriate help added to the care programme to be instituted in their new environment. The suggestion had come initially from the Speech and Language Therapy and Psychology team at the hospital who were working with the clients already and had noted a number of anomalies: for example, some clients appeared to be hearing-impaired but had received no monitoring, whilst others had hearing aids but had not been recently assessed.

The unit's traditional method of dealing with any problems associated with the ears was to refer clients to the doctor in charge on the client's ward, and then, if necessary, to the ENT specialists. This method was to prove inefficient at best, and did not engender a high standard of aural care, as will be discussed later.

It was decided at the outset that special arrangements would be needed to meet the tight demands of the resettlement programme, and the challenging assessment problems that would be created.

\section{CLINIC ARRANGEMENTS}

Careful planning was needed to make the assessments a success, and as there was little or no previously documented experience of such clinics this could not be used to smooth the process. The most important decision concerned the location of the clinics. Our intention was that the assessment process should cause as little disruption as possible to the client. Consequently, the clinics were held in the unit itself, in surroundings that were familiar to the clients. This would help to put them at ease. Occasionally, some clients were seen away from the unit when this was unavoidable. These clients were seen at the local Audiology Department.

A multi-disciplinary team was essential: the audiologist could not perform the necessary tests without the knowledge of the Speech and Language Therapists and Clinical Psychologists, who knew the clients and their abilities. Similarly, the therapists and psychologist did not have the knowledge of audiological techniques that would be used and adapted if the assessments were to be useful. This combination proved to be of great value. Attempts were also made to involve the ward staff, doctors and care workers though the changes of staff and clients due to the closure of the unit meant that longterm support could not be guaranteed. It was also difficult to persuade carers that a hearing problem is one that should be taken seriously. This was further exacerbated by the environment in which the clients lived which has high levels of background noise, which in turn makes care staff use loud voices. This prevents early identification of hearing difficulties.

Most clinics were booked to give each client a specific appointment that would usually last about half an hour, the appointment being sent to the relevant ward at least one month in advance. This was accompanied by a request that the ward doctor should examine the ears beforehand to ensure that they were free of wax and visible disease. Confirmation of this was requested before clinic attendance, but was not made mandatory. A large room with sufficient comfortable chairs for the client, carer and assessment team was used. The chairs were arranged around a table in a rough circle, allowing unrestricted access behind the client, who was seated with the rest of the assessment team in front of him. The room was quiet but not sound-treated, with an ambient noise level below $40 \mathrm{~dB}$ (A).

Equipment was kept to a minimum in order that the client would not be frightened by its presence. At every session an audiometer, tympanometer, otoscope, warble tone generator, rape alarm and other distraction devices were available. Hearing aid repair facilities and impression-taking equipment were also available. All equipment was kept out of sight until needed with the exception of the audiometer when it was to be used for those clients who were familiar with it. 
When the client was brought in by the carer, he was introduced to the assessment team using first names wherever possible. The carer was invited to stay and help with the assessment, especially by discussing their own observations of the client's hearing ability. It was regarded as very important that the client's notes were made available to the assessment team at this stage.

\section{ASSESSMENT}

This was the most important part of the exercise, and the one which caused the most problems. There is little guidance available on test procedures, and it is obvious that no one procedure would be universally acceptable to such a diverse group of clients.

Otological assessment: This was attempted in all clients and was successful in $95 \%$ of cases. The audiologist viewed both tympanic membranes and external auditory canals, recording the results. Other aural or ENT problems were recorded where possible, especially if they needed referral to an ENT surgeon. In addition, examination of the client's notes was undertaken to establish any previous history of ear disease or deafness. The client's carer was questioned as well, but this often proved fruitless as the rapid turnover of staff at this time prevented longterm relationships between clients and carers.

Audiometry: This was possible in a very small group of clients who attended, ie less than $10 \%$. It should be remembered that the client group which was the subject of this survey represented the last $20 \%$ or so of the unit's original population and was potentially the most challenging for a range of physical and behavioural reasons.

Tympanometry: This was used rarely. The main reason for this is the relatively few conductive hearing losses that were found (with the exception of those caused by wax). Where such losses were identified by audiometry or otoscopy, the test was performed to confirm the findings. It was not used as a general method of screening hearing, as is common practice in 'normal' ENT clinics.

Distraction tests: These were used most often and were developed as time went by. The use of a free-field warble tone generator (Cyber Electronics Model CE 70) was a first choice of stimulator. This enabled a variety of high, low and mid-frequency tones to be presented at varying degrees of sound pressure level. Conversational voice was, of course, always available and was supplemented by the availability of home-made stimulators in the form of coins, seeds, ball bearings etc in tins and boxes that could be shaken to create varying levels of sound. The ubiquitous cup and spoon were also used in conjunction with a milk bottle and teapot. Towards the middle of the survey music and an empty biscuit packet were used. A rape alarm was used in extreme cases to allow a stimulus of $110 \mathrm{dBA}$ to be applied.

All of the workers on this project will readily admit that the performance of these tests improved with time and that the early attempts were rather primitive and lacking in finesse. The testing of hearing by such methods has always been so, and the novice usually sits with a recognised expert to learn the necessary techniques as a journeyman, usually in paediatric audiology where such methods are used most often. There are so few similar schemes that this option was denied to the Lancaster team, and it was necessary to develop expertise as the project went along. With that in mind, the team took great care to make sure that the results obtained could be correlated with the other evidence.
The method of distraction testing is well described elsewhere ${ }^{(2)}$ and a detailed description would not be of value here. What is interesting to note is the differences in the technique and the stimuli used when compared to 'conventional' testing. The cup and spoon, beloved of health visitors for testing of babies, became a firm part of the distraction armoury, not because of its place in distraction testing, but because it was soon obvious to the team that the clients would readily respond to sounds that were not only familiar to them, but were also 'beneficial'. The promise of a cup of tea was often the only sound that many would respond to, with the exception of one other - the biscuit packet. This was discovered by accident. The clients often responded to the noise of a packet being opened or crumpled as it may have resulted in a biscuit for them. Other useful but less universal sounds came from a child's story book which had noise generator squares in it. These were designed to be pressed by a child to create the noise of a fire engine, helicopter, train etc. Being electronically generated, they were of a constant output, and sufficiently unusual for many clients to respond. Indeed, it was this latter observation that began to determine the future direction of the testing.

It became apparent that clients would only respond to sounds of the following types:

- beneficial in some way

- totally unfamiliar

- a voiced request in a commanding tone

We were able to show many times that clients would not respond to, say, their names being called in a normal voice, but when a commanding tone of the same sound level was used, a response was elicited.

Thus the essential test stimuli that were used on most clients became the cup and spoon, biscuit wrapper, conversational voice, warble tone generator and any other unusual sounds that could gain a response. From these the team was able to make an assessment of the client's hearing.

Cooperation tests: These are essentially language-based and it was felt that they would be inappropriate for the majority of the clients to be tested. Thus they are not reported.

Objective tests: Two main tests were available. BSER (brain stem evoked responses) could be performed when the client was able to sit quietly whilst the test was performed, or when other procedures (eg dental work) were being carried out under general anaesthetic. OAE (Otoacoustic Emissions) was used as an alternative to BSER and a confirmation of other hearing tests when some doubt existed about their validity. Data for this test, however, is not presented here as it was only introduced toward the end of the data collection period.

\section{RESULTS}

Of all the results that were produced from this survey the one concerning wax was the most significant. Seventy-eight percent of the clients examined had occlusive wax in one or both ears, many after a prior request for wax removal. In many cases the presence of occlusive wax had been reported many years earlier when both the external auditory meati of clients were examined by the doctor conducting the periodic medical examinations. These values are well in excess of those found in the general population, where usually less than $20 \%$ are found to have occlusive wax at attendance for an appointment for hearing aid assessment (private communication to one of the authors). 
The rate of referrals to the ENT department for assessment and treatment is also higher than that found in the normal population $^{(3)}$. It would be difficult to extrapolate this result to the general population because of the low numbers involved in the survey.

Among the conditions found were cholesteatoma, perforation, chronic otitis media and chronic otitis externa.

\begin{tabular}{|lcc|}
\hline & Number & Percentage \\
Non Occlusive Wax & 15 & 21 \\
Bilateral Occlusive Wax & 38 & 54 \\
Unilateral Occlusive Wax & 17 & 24 \\
Referrals to ENT & 6 & 9 \\
\hline
\end{tabular}

Table 1-Ear wax problems

\begin{tabular}{|lccc|}
\hline & Number & Percentage & Mean Age \\
Normal & 46 & 66 & 52 \\
Mild Hearing Loss & 14 & 20 & 53 \\
Moderate Hearing Loss & 8 & 11 & 68 \\
Severe Hearing Loss & 1 & 1 & 43 \\
Profound Hearing Loss & 0 & 0 & - \\
Unknown & 1 & 1 & 34 \\
TOTAL & 70 & $\mathbf{1 0 0 \%}$ & $\mathbf{5 3}$ \\
\hline
\end{tabular}

Table 2 - Levels of hearing loss found

\begin{tabular}{lcc|} 
& Number & Percentage \\
Clients with no recorded history & 44 & 63 \\
Clients with history $<10$ years ago & 1 & 1 \\
Clients with history $10-15$ years ago & 4 & 6 \\
Clients with history 15-20 years ago & 9 & 13 \\
Clients with history $20-25$ years ago & 10 & 14 \\
Clients with history 25-30 years ago & 1 & 1 \\
Clients with history $30-35$ years ago & 1 & 1 \\
\hline
\end{tabular}

Table 3 - Previously reported aural history

The types of hearing loss broadly match those of the normal population with $12 \%$ in need of some form of amplification, compared to the normal population values of $10 \%{ }^{(4)}$. Whilst this seems quite gratifying, the poor sensitivity of the test must be considered. More specific testing may well have shown that some of those classed as normal or mildly impaired have some form of hearing loss suitable for an aid. Surprisingly, in view of the many conditions present which could be associated with deafness, the numbers are not above the normal population average. It is perhaps worth adding that two blind brothers who were not included in this survey have now been discovered to be profoundly deaf, a condition which had gone unrecognised for more than two decades. More tests are scheduled to enable us to gain a better understanding of their residual hearing function.

The number of hearing aids fitted, (seven), was, thankfully, small as the majority did not need any form of amplification. For most clients this was a low-powered hearing aid suitable for mild to moderate hearing losses. In one case a high-powered ear level aid was fitted (an NHS BE 53), indicating a substantial hearing loss had gone unnoticed for a considerable time. Each of the recipients of the hearing aids became part of a care plan designed and operated by the speech therapy staff who allowed gradual introduction of moulds and amplification, initially under strict supervision.

\section{DISCUSSION}

One of the hardest parts of this project was creating and maintaining a suitable testing strategy, whilst overcoming

\begin{tabular}{|lcc|}
\hline & Number & Percentage \\
No Hearing Aid Needed & $62^{*}$ & 89 \\
Hearing Aid Fitted & 7 & 10 \\
Hearing Aid Declined & 1 & 1 \\
*Of these one client was fitted with an auditory & filter to suppress \\
Hyperacusis & & \\
\hline
\end{tabular}

Table 4 - Hearing aid provision

three main hurdles. Firstly, there was no previous experience of testing in similar establishments. Secondly, such tests must be equally applicable to all types of client. Finally, we found that some clients' ability to be distracted by any sound was completely (or almost completely) missing. This was drawn to our attention by the lack of reaction of one client.

We were testing him using the traditional distraction techniques described above and found that he did not respond to any stimuli, even the high intensity rape alarm. Yet he was known to use language and had no reported difficulties with communication on his ward. At the end of the session the audiologist was replacing the equipment (out of the visual field of the client) and accidentally moved a spoon within a teacup. This brought an immediate and correct response from the client, further confirmed by related questions and answers leading to an assessment of normal hearing. After that, the cup and spoon became an integral part of the test battery.

This raised the question whether his lack of response was part of his condition, or part of his conditioning. This lack of response was later observed in many clients of varying degrees of disability and background and led us to form two possible conclusions. Firstly, and most simply, it is a part of his condition which explains why it is not found in all members of this client group. More detailed studies on the aetiology of the conditions would be needed to support this hypothesis. If it is a serious possibility, it raises questions about the validity of the findings of distraction tests performed on learning-disabled children. They may possibly be diagnosed as hearing-impaired and be fitted with a hearing aid because of invalid testing.

The second explanation may well come from an examination of the conditions under which these clients live. Any casual observer will see that it is not a quiet environment. The floors are often covered with a hard surface, and television sets have higher volume levels than would be found in a normal home. This may be due simply to custom, or be used to overcome the higher background noise levels. Firebells often contribute to the general noise and carers' voices are almost always raised, often in a commanding tone. (Many clients would only respond to their own name called in a commanding voice, and did not respond to a normal quality of voice.) The response to sound may be unpredictable or selective. Many of the normal population will achieve similar attitudes to constant extraneous sounds like cars on the road outside our homes at night. The client will successfully block out any extraneous sound which will not be of any immediate benefit as we observed with the rape alarm but not the cup and spoon. Our belief is that these clients will develop an 'auditory window' which allows them to perceive only what is relevant. That window may change in size according to the range of interesting auditory stimuli available. For some it will be wide, whilst for others very narrow, possibly recognising only a single stimulus. Again, this has implications for distraction testing of learningdisabled children.

Our survey also highlighted a number of other problems, the most significant of which is the presence of occlusive 
wax. It is easy to speculate how it occurs with such regularity. It may be due to poor aural management, the dry and dusty atmosphere within such units, or a predilection on the clients' part towards such accumulation. Data on this topic is understandably sparse. It is important that all those being cared for within such establishments should have a regular aural examination, possibly at six-month intervals, to prevent this problem and its associated difficulties for the client. Such an examination would also lead to earlier management of other ENT problems in this client group.

A higher level of awareness of ENT and hearing problems is an essential prerequisite amongst all staff caring for this group. We, like others, have highlighted the number of undiagnosed hearing losses within such a group. This is a very worrying aspect of our findings in a group where good communications are vital, especially when communications programmes are in place.

The need for such a regular examination is further supported by the evidence which we accumulated on previously reported aural problems like otitis externa and perforations. We found $37 \%$ of our population had significant aural problems other than occlusive wax reported in their notes. This is a very significant proportion and certainly much larger than found within the general population referred for hearing aids ${ }^{(3,5)}$. Indeed, it is possible that the numbers may be higher, as not all the clients seen had ear examination reports in their notes.

\section{CONCLUSION}

Our study highlighted the prevalence of hearing and aural disorders in a group of learning-disabled adults, and the need for increased awareness of their auditory status. Many clients remain in this type of care throughout the country, many more have been discharged into the community with similar difficulties, yet few if any are given the benefit of aural assessment.
It is apparent from the data presented that there may be a large number of untreated ear-related disorders. In order to reduce the handicap, training in the identification of hearing loss should be given to all carers who have contact with this group or those in residential care or the community. Such a programme of training and assessment is not without cost, but we suggest that the benefit to a significant percentage of the learning-disabled community would make the investment worthwhile.

\section{ACKNOWLEDGEMENTS}

We would like to extend our sincere thanks to many people, but above all to Keith Fitzsimmons, who was the catalyst for the project; to Craig Jackson, for data analysis; and to Lancaster Priority Services Trust for funding.

\section{REFERENCES}

1 Caring for people. Community care in the next decade and beyond. HMSO 1989

2 McCormick B In: Paediatric Audiology McCormick B (ed) Whurr Publishers Ltd 1993

3 Reeves D et al Direct referral systems for hearing aid provision HMSO 1994

4 Davis AC The prevalence of hearing impairment and reported disability among adults in Great Britain. Int J Epidemiol 1989; 18:911-917

5 Davis AC Epidemiology of hearing disorders In: SconBrown's Otolaryngology 5th ed Butterworth \& Co 1987 90-126

\section{QUIZ}

1. What is an oncogene?

2. What is an anti-oncogene?

3. In hereditary retinoblastoma children usually develop bilateral tumours - why?

4. In sporadic retinoblastoma children always develop unilateral tumours - why?

5. Is hereditary retinoblastoma a dominant or a recessive disease? 